\title{
CREDIT CONTAGION AND AGGREGATE LOSSES
}

\author{
Kay Giesecke \\ Stefan Weber \\ Humboldt-Universität \\ TU Berlin \\ zu Berlin
}

September 10, 2002*

\begin{abstract}
Credit contagion refers to the propagation of economic distress from one firm or sovereign government to another. In this paper we model credit contagion phenomena and study the fluctuation of aggregate credit losses on large portfolios of financial positions. The joint dynamics of firms' credit ratings is modeled by a voter process, which is well-known in the theory of interacting particle systems. We clarify the structure of the equilibrium joint rating distribution using ergodic decomposition. We analyze the quantiles of the portfolio loss distribution and in particular their relation to the degree of model risk. After a proper re-scaling taking care of the heavy tails induced by the contagion dynamics, we provide a normal approximation of both the equilibrium rating distribution and the portfolio loss distribution.
\end{abstract}

Key words: credit contagion, portfolio losses, voter model, Choquet theory, ergodic decomposition, re-scaling

\footnotetext{
${ }^{*}$ Giesecke is at the National Research Center 373, Department of Economics, HumboldtUniversität zu Berlin, Spandauer Str. 1, D-10178 Berlin, Germany, Phone +49 302093 1459, Fax +49 302093 1460, email giesecke@wiwi.hu-berlin.de. Weber is at the Department of Mathematics, TU Berlin, Sekretariat MA 7-4, Str. des 17. Juni 136, 10623 Berlin, Germany, Phone +49 303142 7601, Fax +49 303142 1695, email weber@math.tu-berlin.de. We would like to thank Boris Buchmann, Steffen Dereich and Hans Föllmer for helpful discussions. Giesecke acknowledges financial support by Deutsche Forschungsgemeinschaft via SFB 373. Weber acknowledges financial support by Deutsche Forschungsgemeinschaft via Graduiertenkolleg 'Stochastische Prozesse und Probabilistische Analysis'.
} 


\section{Introduction}

Credit contagion refers to the propagation of economic distress from one firm or sovereign government to another. In this paper we study the fluctuations of aggregate losses due to credit contagion on large portfolios of financial positions.

A number of studies, for example Duffee (1998) and Keenan (2000), have investigated historical default data. They found, quite plausibly, that aggregate default rates are strongly related to general macro-economic factors such as the level of default-free interest rates, GDP growth rates, equity index returns and other business cycle indicators. This sensitivity of firms' financial health on common factors leads to cyclical correlation of defaults. A more direct sort of default dependence arises from direct links across firms, in that the financial distress of one firm can directly trigger the distress of some other firm(s). This 'infectious' propagation or contagion of defaults can develop autonomous dynamics, as the banking crises in Japan and South Korea show. While cyclical dependence between firms corresponds more to 'normal' fluctuation of aggregate default rates, contagious cascading defaults typically result in excessive fluctuations in default rates up to disruption of financial markets. The possibility of disruption corresponds to systemic risk, and would call for intervention of financial market supervising authorities or central banks.

Existing studies on the aggregation of credit risks have exclusively focused on the modeling of cyclical correlations. In these so-called Bernoulli mixture models [Frey \& McNeil (2001)], fluctuations of aggregate losses are due to the fluctuation in some exogenous macro-economic variables. Importantly, conditional on the macro-state variables defaults of different firms and the associated losses are assumed to be independent. Examples include the models put forward by KMV [Kealhofer (1998)], J.P. Morgan [Gupton, Finger \& Bhatia (1997)], and Credit Suisse Financial Products [CSFP (1997)], which have become a standard for credit risk measurement and management in financial institutions. In these approaches, aggregated loss risks are typically measured by some quantile of the distribution of total losses for some horizon. Large-deviations approximations of the tail distribution of total losses were very recently considered by Dembo, Deuschel \& Duffie (2002).

Financial institutions as holders of multiple defaultable claims are particularly vulnerable to excessive fluctuations in default rates and the associated losses on their portfolio positions. Being an integral part of the financial system, a financial institution's exposure to contagious credit quality deterioration of portfolio positions is directly linked to the amount of systemic risk. A thorough 
understanding of the credit contagion process driving aggregated loss risk is therefore essential for the regulation of financial institutions and the efficient control of systemic risk in financial markets. Our contribution aims, for the first time, at assessing the aggregate loss risk on large portfolios of financial positions whose counterparties are subject to credit contagion.

Credit contagion phenomena rest on the existence of proper transmission channels. Direct links between firms in the form of borrowing and lending contracts constitute a typical distress propagation channel. In the banking sector, interbank lending refers to banks' mutual claims, such as overnight and term interbank lending in the Fed funds market or its equivalents. To the extent that interbank loans are neither collateralized nor insured against, the distress of a bank may trigger the subsequent distress of other banks in the lending chain. Here the central bank is forced to intervene in order to stop the contagion process right at the beginning. Rochet \& Tirole (1996) provide a framework for the analysis of systemic risk with respect to interbank lending and central bank policy. Freixas, Parigi \& Rochet (2000) study the stability of the financial system and the coordination role of the financial authorities if an insolvent institution affects the system in various ways depending on the cross-payment pattern in the interbank market. Allen \& Gale (2000) propose an equilibrium model where different sectors of the banking system have overlapping claims on one another in order to buffer liquidity preference shocks. This arrangement is however financially fragile: depending on the degree of connectedness of the buffer system, a small liquidity preference shock in one institution can spread through the economy and cause distress in other institutions as well.

Credit contagion is also a serious concern in manufacturing, where trade credits link suppliers and buyers of goods through a chain of obligations: a firm lends to its customers and borrows from its suppliers. In this sense interfirm trade lending is similar to interbank lending. Kiyotaki \& Moore (1997) develop a model of trade credit and study how a liquidity shock, which leads to distress of an individual customer in the first place, can propagate through the borrowing-lending network and result in a chain reaction in which producers and in turn their suppliers run into financial distress as well.

The aforementioned contributions study simple micro-economic models of direct inter-firm financial linkages and the associated contagion mechanisms. Our intention is, in contrast, to directly consider the consequences of explicit linkages between firms in a descriptive statistical way. In fact, the distress propagation pattern in lending and borrowing networks as established above motivate the contagion dynamics we are going to propose. Specifically, our interaction mechanism is based on the voter-model, which is well-known in the 
theory of interacting particle systems [Liggett (1985), Liggett (1999)]. We suppose that a firm can be in one of two credit rating classes, 'high' or 'low'. The joint evolution of firms' credit ratings over time is modeled by a continuoustime Markov process. Direct interdependence between firms' credit quality is induced through assuming that the transition rate of a given firm's rating is proportional to the number of 'neighboring' firms with opposite rating. That is, the migration probability of a firm's credit quality is explicitly linked to the credit quality status of other neighboring firms. The neighborhood of a specific firm is given by all those firms with which this firm is in some direct business or financial relation. This direct relation provides the transmission channel through which economic distress can be propagated.

We then consider the long-run behavior of the rating process. The structure of the invariant (equilibrium) rating distribution depends on the complexity of the inter-firm connectivity structure in the economy. If the degree of connectedness is low (firms have only few business partners), then the state of an individual firm is highly dominated by connected firms' state. Clusters of firms with the same rating are relatively stable. Their size fluctuates randomly; they grow and merge with other clusters. In the long run only one type of rating appears: either all firms are low-rated, or all firms are high-rated. The probability of these scenarios depends only on firms' marginal credit risk. This implies a high degree of systemic risk. In the micro-economic model of Allen \& Gale (2000), where a liquidity shock faced by an individual bank may be buffered by the interbank-lending network, one obtains qualitatively similar implications. With a simple lending network structure, the buffer capacity is low and a sufficiently severe shock may spread quickly through the banking system and result in bankruptcy of all banks. In this sense a low degree of connectedness corresponds to a high degree of instability and systemic risk in the economy.

With an increasingly complex interdependence structure, the equilibrium rating distribution becomes non-trivial. Random clusters of firms with the same rating appear only locally and their size fluctuates heavily. In particular, they do not merge and grow in the same way as with a less complex network structure, but they are more unstable and less persistent. There are again qualitative parallels to the micro-economic models of Allen \& Gale (2000) and Kiyotaki \& Moore (1997). In the former, if the pattern of inter-connectedness generated by the cross-holdings of claims is complete in the sense that each bank is in a lending relationship with all other banks, the impact of an initial shock may be reduced through an exploitation of the buffer network. In the latter, if the structure of the credit chain is highly complex, i.e. each firm 
has a wide network of business/lending partners, then this arrangement is quite robust against firms in the chain becoming distressed. Here some of the connected firms may act as substitutes for a distressed link in the chain, so that default effects may not be as severe as with few credit relationships.

We investigate the structure of the equilibrium rating distribution in a large complex inter-linked economy, where firms are homogeneous with respect to their marginal credit risk. In the ergodic case, the equilibrium ratings are governed by a so-called extremal distribution corresponding to the fixed degree of marginal credit risk. In the general case the equilibrium ratings are governed by a mixture of extremal rating distributions. The mixing distribution corresponds to the distribution of the average number of low-rated firms in the whole economy, which is a random quantity. The mixing distribution, as well as the expected proportion of low-rated firms, is not changed through the interdependence of firms. What interaction changes is, however, the correlation between firms' ratings. For any finite number of firms the probability to find many firms with the same rating is higher than with independent firms; the equilibrium rating distribution exhibits 'heavy tails'.

In a next step we associate with each firm a random variable describing the loss a financial institution suffers by holding financial positions, such as loans, bonds, stocks, or derivatives, with that firm. The distribution of this loss depends on the rating class to which the firm belongs; conditional on the rating, position losses are assumed to be independent. We then consider aggregate losses on large portfolios of positions, whose interdependent counterparties are subject to credit contagion processes.

Average losses on infinite portfolios are determined through the average proportion of low-rated firms and the expected conditional position losses for both rating classes. While loss uncertainty stemming from the fluctuation of conditional position losses averages out, loss uncertainty remains from the average proportion of low-rated firms, which is in general a random quantity. Average loss randomness is hence governed by the mixing distribution. In the ergodic case the mixing distribution is degenerate and average loss uncertainty vanishes. The same results hold if all interdependence of firms is removed, i.e. in a benchmark economy of independent firms where the probability of a firm to be in the low rating is random and governed by the mixing distribution. We can thus conclude that average losses in infinitely large portfolios are not governed by the interaction of firms.

We show that the quantiles of the distribution of aggregate losses on large portfolios are essentially governed by the quantiles of the mixing distribution. This means that the tail behavior of the distribution of the empirical average 
of low-rated firms essentially determines the tail behavior of aggregate losses, i.e. the extent to which losses on large portfolios fluctuate excessively. This parallels a result of Frey \& McNeil (2001) for Bernoulli mixture models, in which losses are conditionally independent given some set of macro-economic factors. There the tail of the factor distribution essentially determines the tail of the loss distribution.

Using the recent general voter model results of Zähle (2001), we provide an explicit normal approximation for the distribution of total losses on large finite portfolios. This approximation is the key to the measurement and management of the portfolio's aggregated credit risk. Since the contagion dynamics induce heavy tails in the extremal equilibrium rating distribution, the appropriate re-scaling in this approximation is non-classical. Let us emphasize two distinct features of the approximate loss distribution. First, the mixing distribution governs average portfolio losses, which can be random. Second, losses fluctuate around their (possibly random) averages, where the degree of fluctuation depends on the degree of interaction of firms. This effect can most easily be understood in the ergodic case where average portfolio losses are deterministic; in comparison with the benchmark case of independent firms, the loss distribution exhibits heavy tails when firms are interdependent and credit contagion phenomena are present. That is, the probability of exceeding a given loss amount above average losses is larger than with independent firms. The excess probability depends on the complexity of the economy's network structure: the lower the degree of inter-firm connectedness and the higher the degree of firm interaction, the fatter is the tail of the loss distribution, and the higher the likelihood of extreme losses. Also, quite intuitive, the lower the degree of marginal credit risk, the lower is the probability of extreme loss fluctuations.

Empirical studies suggest that there is a close relation between credit contagion, macro-economic shocks, and the degree of systemic risk. In a companion paper Giesecke \& Weber (2002), we consider the distribution of aggregate portfolio losses in the presence of credit contagion phenomena and cyclical default correlations. Alternative and quite different approaches to model cyclical and contagion-like effects have been proposed by Giesecke (2001) and Schönbucher \& Schubert (2001). These contributions focus directly on default events and their evolution over time.

The remainder of this paper is organized as follows. In Section 2 we propose a model for the dynamics of credit contagion. In Section 3, we analyze the asymptotic behavior of the rating process and the structure of the invariant equilibrium rating distribution implied by the contagion model. Losses on large portfolios of positions with interdependent firms are considered in Section 4, 
where we provide an explicit approximation of the distribution of aggregate losses. Section 5 concludes. The appendix contains the proofs.

\section{Modeling Credit Contagion}

In this section we provide a statistical model for the effects of credit contagion phenomena as we discussed them in the Introduction. In contrast to the microeconomic approaches, we describe the interactions of the firms probabilistically and consider their consequences on the level of both the whole economy and large portfolios. Based on the discussed propagation pattern, we postulate a basic voter process for the dynamics of the contagion process. The voter model is well-known in the theory of interacting particle systems [Liggett (1985), Liggett (1999)].

We consider an economy with a collection $S$ of firms. A firm $i \in S$ can be in two states, denoted 0 and 1 . State 0 corresponds to a high credit rating and a low default probability, while state 1 corresponds to a low credit rating and a high default probability. The state of the economy is characterized by a configuration in the state space $\{0,1\}^{S}$. We are interested in the evolution of firms' credit ratings over time and the interdependence of the ratings of different firms.

Motivated by our discussion in the Introduction, we assume that the evolution of the rating of an arbitrary firm $i$ is influenced by the state of a collection $\mathcal{N}(i) \subseteq S \backslash\{i\}$ of business partners. $\mathcal{N}(i)$ will be called the set of neighbors of firm $i$. For simplicity, we assume that firms influence each other in a symmetric way: if firm $i$ 's rating is influenced by firm $j$, then firm $j$ 's rating is influenced by firm $i$. Expressed in terms of the neighborhoods, this means:

$$
j \in \mathcal{N}(i) \Rightarrow i \in \mathcal{N}(j)
$$

If we connect all firms $i \in S$ to their neighbors $j \in \mathcal{N}(i)$, we get an undirected graph which characterizes the business relations of the firms. For tractability, we assume a simple neighborhood structure which is specified by a $d$-dimensional lattice. In particular, all firms have the same finite number of business partners.

Hence, we consider an economy with a countably infinite number of firms. Firms are identified with their location on the $d$-dimensional integer lattice $S=$ $\mathbb{Z}^{d}$. The evolution of firms' credit rating over time is described by a continuoustime Feller process $\left(\eta_{t}\right)_{t \geq 0}$ with state space $X=\{0,1\}^{\mathbb{Z}^{d}}$ and transition rate $c$ 
given by

$$
c(i, \xi)=\left\{\begin{array}{rll}
\frac{1}{2 d} \sum_{j:|i-j|=1} \xi(j) & \text { if } \quad \xi(i)=0 \\
\frac{1}{2 d} \sum_{j:|i-j|=1}[1-\xi(j)] & \text { if } \quad \xi(i)=1
\end{array}\right.
$$

That is, a firm $i \in \mathbb{Z}^{d}$ with a high rating $(\xi(i)=0)$ migrates to a low rating $(\xi(i)=1)$ at a rate proportional to the number of low-rated neighboring firms $j \in\{j: \xi(j)=1,|i-j|=1\}$, and vice versa. It is easy to see that if all firms $i \in \mathbb{Z}^{d}$ are either in good or in bad shape, then the transition rate is zero. Put another way, after a unit exponential waiting time in one state, a firm $i \in \mathbb{Z}^{d}$ migrates to the state of some neighboring firm $j \in\{j$ : $|i-j|=1\}$ which is chosen with probability $1 / 2 d$. A rating transition is hence a Poisson event, whose intensity is proportional to the number of neighboring firms with opposite rating. In this sense our credit contagion model belongs to the class of intensity based credit risk models [see, e.g., Jarrow \& Turnbull (1995), Duffie, Schroder \& Skiadas (1996), Duffie \& Singleton (1999), Jarrow, Lando \& Turnbull (1997), and Lando (1998) for single-firm models]. The idea that some firm's intensity of default may also directly depend on the state of other firms in the economy has recently appeared in Jarrow \& Yu (2001) and Davis \& Lo (2001).

This formal model of firms' joint credit migration probabilistically describes the pattern of credit contagion phenomena as we discussed them in the Introduction. Let us pick the specific example of trade credit. If a highly rated firm's business partners in a trade credit (the 'neighboring' firms) are distressed, i.e. have the low credit rating, then the probability that this firm runs into distress as well (i.e. migrates to the low rating) due to a payment default in the credit chain increases with the number of distressed partners. If a distressed firm's business partners in the credit chain are in good shape (and hence a default in the chain is unlikely), then the probability of that firm's recovery from distress increases with the number of healthy partners.

\section{Equilibrium Behavior}

Let us now look at the equilibrium distributions and the asymptotic behavior of $\eta$. It turns out that the structure of the equilibrium distributions depends on the dimension $d$. The dimension $d$ can be interpreted as the degree of connectedness of firms in the economy; with increasing $d$ the structure of inter-firm connections becomes more complex. The larger $d$, the more business partners has any individual firm. At the same time the number of indirect inter-firm 
links of given length increases. More specifically, if $i$ and $j$ are two firms, a sequence $\left(i_{0}, i_{1}, \ldots, i_{n}\right)$ of firms is a link of length $n$ between $i$ and $j$, if $i_{k}$ is a neighbor of $i_{k+1}$ for $k \in\{0,1, \ldots, n-1\}, i_{0}=i$ and $i_{n}=j$. The number of links of length $n$ between a given firm and some other firm equals $2^{d} \cdot\left(2^{d}-1\right)^{n-1}$ and grows exponentially in $n$ and $d$.

\subsection{Low Degree of Inter-Firm Connectedness}

Let $\mu$ denote the initial distribution of $\eta$. The process $\eta$ has càdlàg paths; for convenience, we will work with the canonical version of the process. $\Omega$ denotes the space of càdlàg functions on $\mathbb{R}_{+}$with values in $X$ endowed with the usual augmented filtration. For the law of the process $\eta$ we will write $P^{\mu}$.

We shall assume that $\mu$ is translation-invariant and denote by

$$
\rho=\mu\{\xi: \xi(i)=1\}
$$

the Bernoulli parameter of the initial marginal rating distribution for an arbitrary firm $i . \rho$ can hence be thought of as a measure of an individual firm's marginal credit risk. With respect to this marginal risk the firms in the economy are homogeneous. For $d=1,2$ and translation-invariant initial law $\mu$, as $t \rightarrow \infty$ the distribution of $\eta_{t}$ converges weakly to the mixture

$$
\rho \delta_{1}+(1-\rho) \delta_{0}
$$

cf. Liggett (1999). $\delta_{\xi}$ is the Dirac measure placing mass 1 on configuration $\xi \in X$; in (2) the indices 0 resp. 1 refer to the configurations with all firms being of good resp. bad type. The process of ratings $\eta$ clusters, i.e. for all $i, j \in \mathbb{Z}^{d}$ we have

$$
\lim _{t \rightarrow \infty} P^{\mu}\left[\eta_{t}(i) \neq \eta_{t}(j)\right]=0
$$

For a low degree of connectedness between firms $(d \leq 2)$, in the long run $t \rightarrow \infty$ only one type of rating appears: with probability $\rho$ all firms are lowly rated, and with probability $1-\rho$ all firms are highly rated. The economy ends up in one of these two possible extreme scenarios. The marginal rating distribution of any individual firm is invariant under the contagion dynamics: the degree of marginal credit risk is not affected by the interaction process. Nevertheless, the economy can change drastically on the macroscopic level.

This behavior is quite intuitive in the trade credit chain interpretation. If initially the marginal probability $\rho$ of individual firms to have the low rating is high, then it is quite likely that healthy firms in the credit chain become 
infected. Random clusters of firms with low ratings emerge with high probability, while clusters of firms with high ratings emerge only with low probability. In any case, if the chain a firm operates in is 'short', then the state of the relatively few business partners highly dominates the state of a firm in the chain and clusters of firms of the same type are relatively stable. The size of the clusters changes through random fluctuations, but for low dimensions $d \leq 2$ some of the clusters merge and form large growing clusters. Asymptotically, with high probability $\rho$ all firms are in the bad state, and with low probability $1-\rho$ all firms are in the good state. Vice versa, if $\rho$ is low, then it is unlikely that a firm gets distressed. In the limit, with high probability $1-\rho$ all firms will have the good rating, with low probability $\rho$ the bad rating.

\subsection{High Degree of Inter-Firm Connectedness}

The limiting behavior of $\eta$ differs for higher dimensions $d>2$. In this case, for any translation-invariant ergodic initial distribution $\mu$ with

$$
\rho=\mu\{\xi: \xi(i)=1\}
$$

as $t \rightarrow \infty$ the distribution of $\eta_{t}$ converges weakly to the non-trivial extremal invariant measure $\nu_{\rho}$ of the voter model in dimension $d$ with parameter

$$
\rho=\nu_{\rho}\{\xi: \xi(i)=1\}
$$

cf. Liggett (1999). In this case the rating process $\eta$ coexists, referring to the lack of rating clustering in the long run. If there is a high degree of connectedness in the economy, then the credit contagion process is non-trivial and the long-run ratings are distributed according to $\nu_{\rho}$. Random clusters of firms of equal rating do not grow in the same way as we observed in the case $d \leq 2$. In contrast, for high degrees of connectedness the empirical average of the number of low-rated firms is a preserved quantity under the dynamics and equals $\rho$ forever.

Let us now study the equilibrium rating distribution in case $d>2$ for general, i.e. not necessarily ergodic, translation invariant initial rating distributions. By $\mathcal{M}_{e}$ we denote the space of ergodic probability measures on $X$ endowed with weak topology. We write $\mathcal{M}_{e, \rho}$ for the closed subspace of $\mathcal{M}_{e}$ of probability measures $\nu$ with $\nu\{\xi: \xi(i)=1\}=\rho \in[0,1]$. For any translation invariant probability measure $\mu$ on $X$, the theorem of Choquet states that there exists a probability measure $\gamma$ on $\mathcal{M}_{e}$ such that

$$
\mu=\int_{\mathcal{M}_{e}} \nu \gamma(d \nu)
$$


so that $\mu$ can be represented as a mixture of ergodic measures $\nu$. In Theorem A.2 in the Appendix we prove a refined Choquet decomposition which can be used to establish the complete convergence theorem for $\eta$ in case $d>2$ for general translation invariant initial rating distributions:

Theorem 3.1. Let $d>2$ and denote by $\mu_{t}^{\kappa}$ the distribution of $\eta_{t}$ for given initial distribution $\kappa$ on $X$. Let $\kappa$ be a translation invariant measure, and let

$$
\kappa=\int_{[0,1]}\left(\int_{\mathcal{M}_{e}} \nu \gamma_{\rho}(d \nu)\right) Q(d \rho)
$$

be the refined ergodic decomposition of $\kappa$, cf. Theorem A.2. Then we have that

$$
\mu_{t}^{\kappa}=\int_{[0,1]}\left(\int_{\mathcal{M}_{e}} \mu_{t}^{\nu} \gamma_{\rho}(d \nu)\right) Q(d \rho),
$$

and

$$
\mu_{t}^{\kappa} \Longrightarrow \int_{[0,1]} \nu_{\rho} Q(d \rho)
$$

where $\nu_{\rho}$ is the extremal invariant measure of the basic voter model in dimension $d>2$ with parameter $\rho \in[0,1]$.

The refined ergodic decomposition (7) describes the initial rating distribution $\kappa$ as a two-step random process: first the parameter $\rho \in[0,1]$ is chosen according to the distribution $Q$, which then prescribes the translation invariant regime

$$
\kappa_{\rho}:=\kappa_{\rho, 0}:=\int_{\mathcal{M}_{e}} \nu \gamma_{\rho}(d \nu) .
$$

The distribution $Q$ governs the mixture of the regimes $\kappa_{\rho}$ in the decomposition of the initial distribution.

The effect of the evolution of the rating distribution in time is described by (8) and (9). If the initial distribution $\kappa$ can be decomposed as in (7), then the rating distributions $\mu_{t}^{\kappa}$ at time $t$ and $\mu_{\infty}^{\kappa}=\lim _{t \rightarrow \infty} \mu_{t}^{\kappa}$ can be decomposed analogously. Theorem 3.1 describes these rating distributions as two-step random processes: first the parameter $\rho \in[0,1]$ is chosen according to the distribution $Q$, which then determines the regimes

$$
\kappa_{\rho, t}=\left\{\begin{array}{ll}
\int_{\mathcal{M}_{e}} \mu_{t}^{\nu} \gamma_{\rho}(d \nu) & \text { if } t<\infty \\
\nu_{\rho} & \text { if } t=\infty
\end{array} .\right.
$$


An understanding of the decompositions

$$
\mu_{t}^{\kappa}=\int_{[0,1]} \kappa_{\rho, t} Q(d \rho)
$$

for $t \in[0, \infty]$ requires therefore a characterization of the common properties of $\kappa_{\rho, t}$ for a given $\rho \in[0,1]$ and varying $t$. Such a characterization can be provided in terms of the average proportion of firms in the rating classes.

First observe that for translation invariant rating distributions, the empirical average of low-rated firms in the whole economy converges almost surely to a random variable, i.e.

$$
\lim _{n \rightarrow \infty}\left|\Lambda_{n}\right|^{-1} \sum_{i \in \Lambda_{n}} \xi(i)=\bar{\rho}
$$

where $\Lambda_{n}=[-n, n]^{d}$. In general, $\bar{\rho}$ is not deterministic, but random. The regimes $\kappa_{\rho, t}$ have the special property that $\bar{\rho}$ is a.s. deterministic.

Corollary 3.2. Under the assumptions of Theorem 3.1, we can characterize the behavior of $\bar{\rho}$ as follows.

- The average proportion of low-rated firms $\bar{\rho}$ is $\kappa_{\rho, t}$-almost surely equal to $\rho$ for $\rho \in[0,1]$ and $t \in[0, \infty]$.

- For $t \in[0, \infty]$ the law of $\bar{\rho}$ under $\mu_{t}^{\kappa}$ equals $Q$.

This result is a direct consequence of the ergodic decomposition: For ergodic measures $\nu \in \mathcal{M}_{e, \rho}, \bar{\rho}$ is $\nu$-almost surely constant and equal to the parameter $\rho$. This property is invariant under the contagion dynamics, i.e. $\bar{\rho}$ is $\mu_{t}^{\nu}$-almost surely constant and equal to the parameter $\rho$ for given $\nu \in \mathcal{M}_{e, \rho}$ and for arbitrary $t \in[0, \infty]$. Since each measure $\gamma_{\rho}(\rho \in[0,1])$ associated with $\kappa$ is concentrated on the ergodic measures $\mathcal{M}_{e, \rho}$, the behavior of $\bar{\rho}$ under $\mu_{t}^{\nu}$ is inherited by the mixtures $\kappa_{\rho, t}$. Hence, the average proportion of low-rated firms $\bar{\rho}$ is $\kappa_{\rho, t}$-almost surely equal to $\rho$ for $\rho \in[0,1]$ and $t \in[0, \infty]$.

As discussed above, the rating distribution $\mu_{t}^{\kappa}$ can be interpreted as a two-step random process where the regime $\kappa_{\rho, t}$ is chosen according to the distribution $Q$. This implies that the law of $\bar{\rho}$ under $\mu_{t}^{\kappa}$ equals $Q$, as stated in Corollary 3.2. The law of the average number of low-rated firms in the economy is preserved under the contagion dynamics; it is not changed through the interdependence of firms. What interaction between firms changes is the correlation between the ratings of different firms. For any finite number of firms, the probability to find many firms in the same rating class is higher than 
in the case of independent firms; the equilibrium rating distribution exhibits 'heavy tails'.

Observe the following consequence for the equilibrium rating distribution which is of particular importance: Asymptotically, the rating distribution $\mu_{\infty}^{\kappa}$ is a probability-weighted average of extremal invariant measures $\nu_{\rho}$ of the voter model; this mixture is governed by the distribution $Q$ which is given by the initial law of the average number $\bar{\rho}$ of low-rated firms in the economy.

\section{Aggregate Losses on Large Portfolios}

In the previous section we modeled credit contagion phenomena induced by the interdependence of firms and analyzed the weak convergence of the rating process $\eta$ and its distribution in equilibrium. In this section we consider the aggregate losses associated with credit contagion phenomena. Throughout, we suppose that the economy is in equilibrium, in the sense that the distribution of firms' ratings is invariant.

Consider a financial institution holding some portfolio of financial positions whose market value is subject to the credit quality of the issuer or counterparty. Such positions can include not only loans, bonds, or other debt instruments, but also derivatives written by default-prone counterparties. Due to adverse changes in the credit quality of counterparties, for example credit rating downgrades, the market valuation of the corresponding positions can be severely reduced. Suppose the institution holds positions with firms $i \in \Lambda_{n} \subseteq$ $\mathbb{Z}^{d}$, where $\Lambda_{n}=[-n, n]^{d}$. The parameter $n \in \mathbb{N}$ determines the size of the portfolio $\Lambda_{n}$. Risk measurement aims at evaluating the potential losses induced by credit quality deterioration of firms in portfolio $\Lambda_{n}$ at some fixed time horizon. Denoting the losses on positions contracted with firm $i \in \Lambda_{n}$ by the random variable $U(i)$, one is interested in the distribution of aggregate losses

$$
L_{n}=\sum_{i \in \Lambda_{n}} U(i)
$$

We make the following assumptions. Conditional on the rating $r \in\{0,1\}$ of a firm, losses are independent. The conditional distribution $M_{r}$ of losses with respect to a firm having rating $r$ depends only on $r$. We suppose that losses are supported in a bounded interval on $\mathbb{R}_{+}$. We take $M_{r}$ as given and let $l_{r}=\int w M_{r}(d w)$ denote the expected loss caused by a firm of rating type $r$. Clearly, for highly rated firms the probability of (large) losses is small relative to firms in the low rating class. $M_{1}$ is more concentrated on large values than $M_{0}$. Specifically, we might assume that $M_{1}$ stochastically dominates $M_{0}$, i.e. 
for all bounded increasing functions $f: \mathbb{R}_{+} \rightarrow \mathbb{R}: \int f d M_{1} \geq \int f d M_{0}$. We however only suppose that $l_{1}>l_{0}$.

Throughout, we will focus on the realistic situation in which the economy exhibits a high degree of connectedness, i.e. the structure of inter-firm linkages is quite complex and $d>2$.

\subsection{Deterministic Conditional Losses}

We begin our analysis of aggregate portfolio losses $L_{n}$ in this section under the simplifying assumption that credit losses $U(i)$ depend deterministically on the rating class of firm $i$. Specifically, we simply set $M_{r}=\delta_{r}$ for $r \in\{0,1\}$. This implies that the institution suffers no loss from positions with highly rated firms (where $r=0$ ), and a loss of one unit of account from positions with firms having the low rating $(r=1)$.

Let $\mu=\int_{0}^{1} \nu_{\rho} Q(d \rho)$ be an equilibrium rating distribution. Consider the average loss $\left|\Lambda_{n}\right|^{-1} L_{n}$ in portfolio $\Lambda_{n}$. By a law of large numbers,

$$
\lim _{n \rightarrow \infty} \frac{L_{n}}{\left|\Lambda_{n}\right|}=\rho
$$

$\mu$-almost surely, where $\rho$ is random with distribution $Q$. That is, even with deterministic conditional loss amounts not all loss uncertainty averages out. There is still uncertainty concerning average portfolio losses which is governed by the distribution $Q$. As discussed, only if the initial rating distribution is a mixture of ergodic measures $\nu \in \mathcal{M}_{e, \rho}$ for some fixed $\rho \in[0,1]$ (or simply $Q=\delta_{\rho}$ ), this uncertainty vanishes. Since

$$
\frac{L_{n}}{\left|\Lambda_{n}\right|}=\frac{1}{\left|\Lambda_{n}\right|} \sum_{i \in \Lambda_{n}} U(i)=\frac{1}{\left|\Lambda_{n}\right|} \sum_{i \in \Lambda_{n}} \xi(i),
$$

in this case we can identify $\rho \mu$-a.s as the average proportion of low-rated firms in large portfolios when taking the limit $n \rightarrow \infty$ (we adopted this interpretation already in Section 3).

It is important to observe that, due to the ergodicity of the measures $\nu_{\rho}$, the average portfolio loss is not governed by the interaction of the firms, but simply by the distribution $Q$. Let us compare this with the benchmark case of independent firms having the same marginal rating distribution. If we replace $\nu_{\rho}$ by a product measure $\pi_{\rho}$ of Bernoulli distributions with parameter $\rho$ and consider a rating distribution $\hat{\mu}=\int_{0}^{1} \pi_{\rho} Q(d \rho)$, equation (11) still holds $\hat{\mu}$-almost surely - despite the fact that contagion is not present any more. The mixture $\hat{\mu}$ corresponds to an economy in which the ratings of individual firms 
are not interdependent, but where the marginal probability of individual firms to have a low rating is uncertain and distributed according to $Q$.

Let us return to the situation with contagion. We start with the special case where $Q=\delta_{\rho}$ for $\rho \in(0,1)$ and investigate the portfolio losses associated with the extremal invariant credit rating distribution $\nu_{\rho}$. The case of general $Q$ is considered later.

Theorem 4.1. Let $d>2$ and $Q=\delta_{\rho}$ for $\rho \in(0,1)$. Suppose additionally that $M_{r}=\delta_{r}$ for $r \in\{0,1\}$. For large portfolios the law of the losses $L_{n}$ can be approximated by a normal distribution:

$$
\left|\Lambda_{n}\right|^{-\frac{d+2}{2 d}} \cdot\left(L_{n}-\left|\Lambda_{n}\right| \cdot \rho\right)=\left|\Lambda_{n}\right|^{-\frac{d+2}{2 d}} \cdot \sum_{i \in \Lambda_{n}}(\xi(i)-\rho) \Longrightarrow \mathcal{N}\left(0, \sigma^{2}\right),
$$

where the limiting variance $\sigma^{2}=\sigma^{2}(d)$ is given by

$$
\sigma^{2}=\rho(1-\rho) \cdot \frac{\gamma_{d} \cdot d}{2^{d+3} \pi^{d / 2}} \cdot \Gamma\left(\frac{d-2}{2}\right) \cdot \int_{[-1,1]^{d}} \int_{[-1,1]^{d}} \frac{1}{\|x-y\|_{2}^{d-2}} d x d y,
$$

where $\Gamma$ is the Gamma-function and $\gamma=\gamma_{d}$ is given by

$$
\frac{1}{\gamma}=(2 \pi)^{-d} \int_{(-\pi, \pi)^{d}}\left(1-\frac{1}{d} \sum_{m=1}^{d} \cos x_{m}\right)^{-1} d x .
$$

The loss distribution can uniformly be approximated:

$$
\sup _{x \in \mathbb{R}_{+}}\left|\nu_{\rho}\left(L_{n} \geq x\right)-\Phi\left(\frac{\left|\Lambda_{n}\right|^{1 / 2} \rho-\left|\Lambda_{n}\right|^{-1 / 2} x}{\sigma \cdot\left|\Lambda_{n}\right|^{1 / d}}\right)\right| \leq \epsilon_{n}
$$

where $\epsilon_{n} \rightarrow 0$ as $n \rightarrow \infty$, and $\Phi$ is the standard normal distribution function.

We emphasize that the re-scaling in (13) is non-classical. This is due to the heavy tails in the equilibrium rating distribution, which result from the contagion dynamics. Unfortunately, we are not able to provide bounds of BerryEsseen-type for the errors $\epsilon_{n}$ in (16), which would help to understand the speed of convergence.

By inequality (16) the probability of a loss larger than $x \in \mathbb{R}_{+}$can uniformly be approximated by the function

$$
\Psi_{d, \rho}\left(\left|\Lambda_{n}\right|, x\right)=\Phi\left(\frac{\left|\Lambda_{n}\right|^{1 / 2} \rho-\left|\Lambda_{n}\right|^{-1 / 2} x}{\sigma(d) \cdot\left|\Lambda_{n}\right|^{1 / d}}\right)
$$

where $\left|\Lambda_{n}\right|=(2 n+1)^{d}$ is the size of the portfolio $\Lambda_{n}=[-n, n]^{d}$. Heuristically, interpolation between sizes of the portfolios $\Lambda_{n}$ allows us to define the approximate loss probabilities larger than $x \in \mathbb{R}_{+}$for portfolio size $r \in \mathbb{R}_{+}$by

$$
\Psi_{d, \rho}(r, x)=\Phi\left(\frac{r^{1 / 2} \rho-r^{-1 / 2} x}{\sigma(d) \cdot r^{1 / d}}\right) .
$$


The interaction of the firms leads to strong correlations of the ratings of different firms. We shall compare the results for the rating distribution $\nu_{\rho}$ to the benchmark case of independent firms which have the same marginal rating distribution. That is, we will assume that the benchmark rating distribution $\pi_{\rho}$ of the firms is given by a product of Bernoulli measures with density $\rho$. If we exchange $\nu_{\rho}$ against $\pi_{\rho}$, we have to replace the normalization factor $\left|\Lambda_{n}\right|^{-\frac{d+2}{2 d}}$ in (13) simply by the usual $\left|\Lambda_{n}\right|^{-\frac{1}{2}}$ and use instead of the limiting variance $\sigma^{2}$ the quantity $\rho(1-\rho)$. The uniform approximation (16) becomes in this case

$$
\sup _{x \in \mathbb{R}_{+}}\left|\pi_{\rho}\left(L_{n} \geq x\right)-\Phi\left(\frac{\left|\Lambda_{n}\right|^{1 / 2} \rho-\left|\Lambda_{n}\right|^{-1 / 2} x}{\sqrt{\rho(1-\rho)}}\right)\right| \leq \epsilon_{n},
$$

where $\epsilon_{n} \rightarrow 0$ as $n \rightarrow \infty$. For independent firms the speed of convergence to the normal distribution can be bounded by the Berry-Esseen theorem (see e.g. Theorem 4.9. and Remarks in Chapter 2 of Durrett (1996)):

$$
\epsilon_{n} \leq \frac{1+2 \rho(\rho-1)}{\sqrt{\rho(1-\rho)}} \cdot \frac{1}{(2 n)^{d / 2}}
$$

By inequality (19) the probability of a loss larger than $x \in \mathbb{R}_{+}$can uniformly be approximated by the function

$$
\hat{\Psi}_{\rho}\left(\left|\Lambda_{n}\right|, x\right)=\Phi\left(\frac{\left|\Lambda_{n}\right|^{1 / 2} \rho-\left|\Lambda_{n}\right|^{-1 / 2} x}{\sqrt{\rho(1-\rho)}}\right) .
$$

Again interpolation between sizes of the portfolios $\Lambda_{n}$ allows us to define the approximate loss probabilities larger than $x \in \mathbb{R}_{+}$for portfolio size $r \in \mathbb{R}_{+}$by

$$
\hat{\Psi}_{\rho}(r, x)=\Phi\left(\frac{r^{1 / 2} \rho-r^{-1 / 2} x}{\sqrt{\rho(1-\rho)}}\right) .
$$

For illustration, let us consider a portfolio of size $r=10.000$ and a marginal rating distribution with parameter $\rho=0.5$, i.e. the marginal probability that a firm has a low rating is 0.5. In Figures 1 and 2 we plot the approximate loss distribution for the benchmark case and the interaction case, where for the latter case we vary the degree $d$ of inter-firm connectedness. As expected, in comparison with the independence case the loss distribution exhibits heavy tails when firms are inter-connected and credit contagion phenomena are present. Put another way, firm interdependence leads to the portfolio being more risky in terms of large losses: with interdependence, the probability of exceeding a given loss amount above average losses is larger than in the independence (benchmark) case. We observe that the degree of 


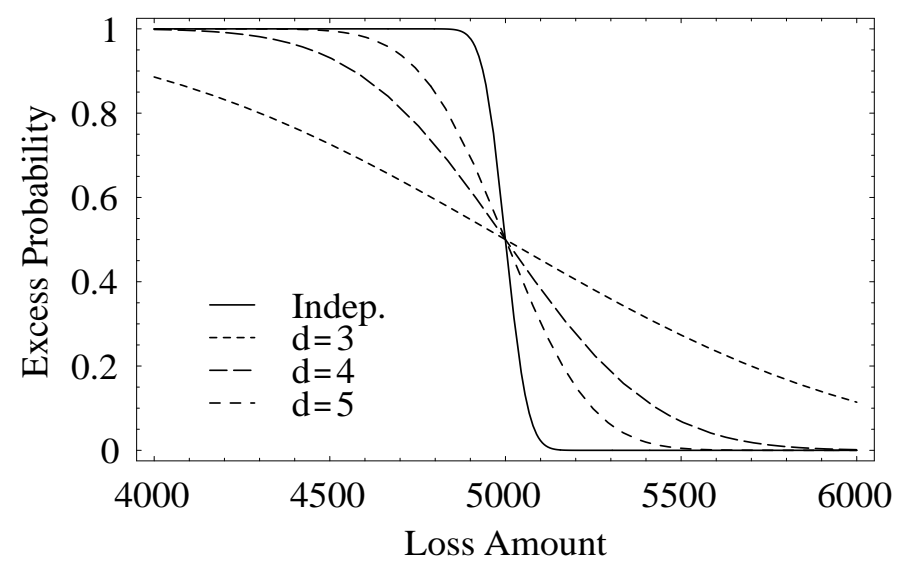

Figure 1: Probability of a portfolio loss exceeding a given amount, varying the degree $d$ of connectedness $(r=10.000$ and $\rho=0.5)$.

differences in loss probabilities depends on the complexity of the economy's network structure: the lower the degree of inter-firm connectedness, the fatter is the tail of the loss distribution. In this sense, the economy with the lowest degree $d$ of connectivity (here $d=3$ ) induces the riskiest portfolios. The higher $d$, the thinner the tail and the lower the likelihood of extreme losses. The underlying idea of this effect was already stressed in Section 3.2: the more complex the economy's connectivity structure, the lower is the degree of firm interaction (the lower is the probability of observing persistent and growing clusters of firms of equal rating), and therefore the less likely is an extreme loss in a large portfolio of firms. The appearing non-trivial equilibrium rating distribution depends on the marginal credit risk $\rho$, and so does the loss distribution. The dependence of loss probabilities on $\rho$ for a given $d$ is shown in Figure 3: the lower $\rho$, the lower is the likelihood of suffering extreme losses in a large portfolio.

The approximate loss density for benchmark and interaction case (in dependence of $d$ ) is shown in Figure 4 . While in case $Q=\delta_{\rho}$ all loss uncertainty averages out in infinite portfolios (cf. (11)), for finite portfolios losses fluctuate around $r \cdot \rho=5000$. Corresponding to our discussion above, with interdependent ratings losses fluctuate more excessively when compared with the benchmark case, where the degree of fluctuation decreases with increasing degree $d$ of connectedness.

Having investigated the loss distribution in the special case where $Q=\delta_{\rho}$ 


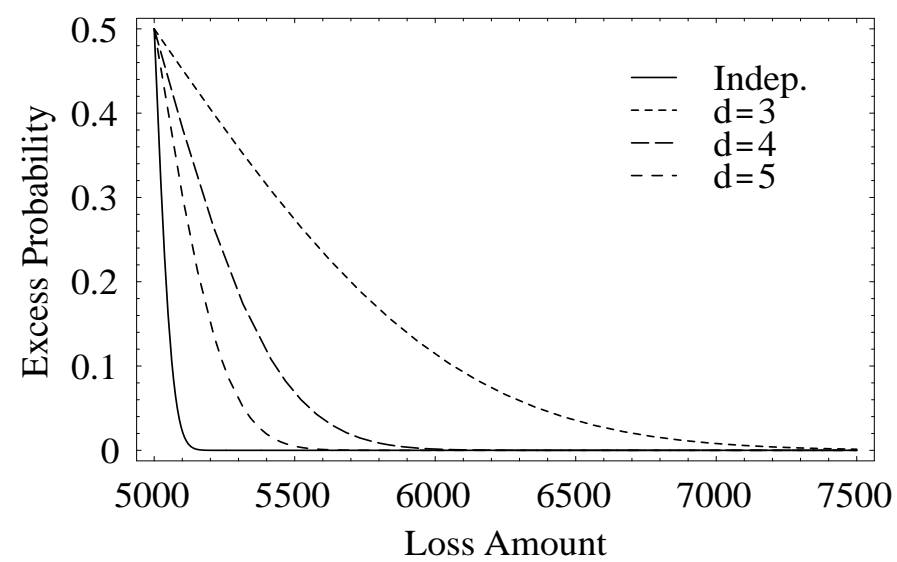

Figure 2: Probability of a portfolio loss exceeding a given amount, varying the degree $d$ of connectedness $(r=10.000$ and $\rho=0.5)$.

for $\rho \in(0,1)$, we now consider the case of general $Q$. In this situation the invariant rating distributions $\mu$ are mixtures of the extremal measures $\nu_{\rho}$, which we focused on in the special case (for a given $\rho$ ). Let $\mu=\int_{0}^{1} \nu_{\rho} Q(d \rho)$ be an equilibrium rating distribution. If $Q$ puts positive mass on 0 or 1 , all firms have the same rating with positive probability. In order to avoid unnecessary technical complications, we exclude these trivial cases as before and assume $Q(\{0\})=Q(\{1\})=0$. In this general case, the exact probability of a loss larger than $x \in \mathbb{R}_{+}$equals

$$
\int \nu_{\rho}\left(L_{n} \geq x\right) Q(d \rho)
$$

In a large portfolio, the law of the losses $L_{n}$ can be uniformly approximated by a mixture of normal distributions:

Corollary 4.2. Let $d>2$ and $M_{r}=\delta_{r}$ for $r \in\{0,1\}$. The distribution of portfolio losses $L_{n}$ can uniformly be approximated, i.e.

$$
\sup _{x \in \mathbb{R}}\left|\int \nu_{\rho}\left(L_{n} \geq x\right) Q(d \rho)-\int \Phi\left(\frac{\left|\Lambda_{n}\right|^{1 / 2} \rho-\left|\Lambda_{n}\right|^{-1 / 2} x}{\sigma(\rho)\left|\Lambda_{n}\right|^{1 / d}}\right) Q(d \rho)\right| \leq \epsilon_{n},
$$

where the error bound $\epsilon_{n} \rightarrow 0$ as $n \rightarrow \infty$.

Based on this result, in close analogy to (18) interpolation between sizes of the portfolios $\Lambda_{n}$ allows us to define the approximate loss probabilities larger 


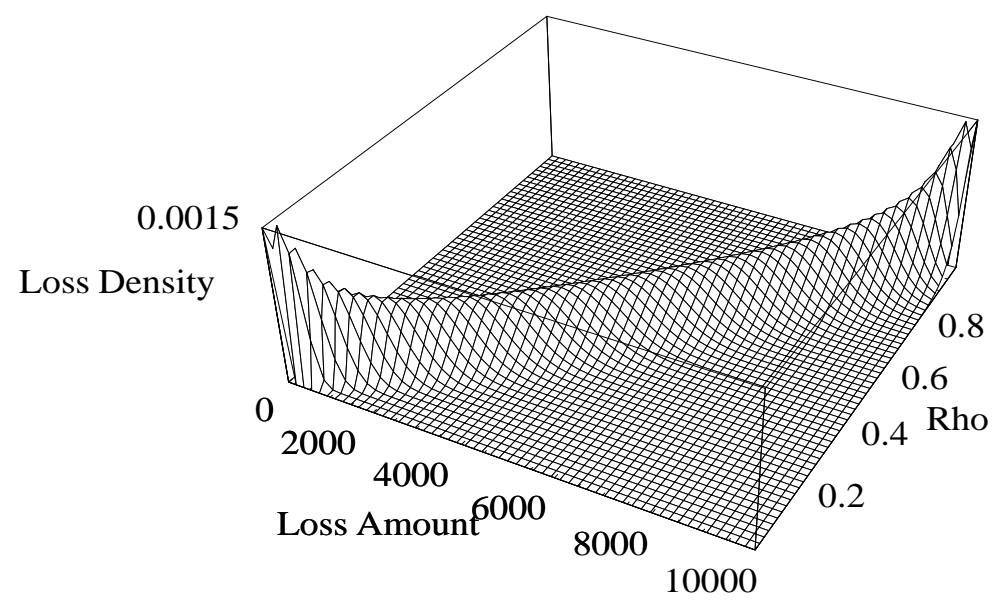

Figure 3: Approximate density of portfolio losses, varying the marginal rating distribution $\rho(r=10.000$ and $d=3)$.

than $x \in \mathbb{R}_{+}$for portfolio size $r \in \mathbb{R}_{+}$by

$$
\int \Phi\left(\frac{r^{1 / 2} \rho-r^{-1 / 2} x}{\sigma(\rho) \cdot r^{1 / d}}\right) Q(d \rho)
$$

Paralleling (22), in the benchmark case with independent firms the approximate loss probabilities can be defined by

$$
\int \Phi\left(\frac{r^{1 / 2} \rho-r^{-1 / 2} x}{\sqrt{\rho(1-\rho)}}\right) Q(d \rho), \quad x, r \in \mathbb{R}_{+} .
$$

In Figure 5 we illustrate the approximate density of portfolio losses in case $Q=0.4 \delta_{0.3}+0.6 \delta_{0.7}$. The portfolio size is again $r=10.000$. In infinite portfolios, according to the distribution $Q$ average losses $\rho=0.3$ with probability 0.4 and $\rho=0.7$ with probability 0.6 . In finite portfolios losses fluctuate substantially around $0.3 \cdot r=3000$ (with probability 0.4 ) and $0.7 \cdot r=7000$ (with probability 0.6 ), as prescribed by $Q$. In analogy to the (no-uncertainty) case $Q=\delta_{\rho}$ considered in Figure 4, interaction leads to more excessive fluctuations when compared to the benchmark case, where the degree of fluctuation depends on the connectivity structure of the economy.

\subsection{Stochastic Conditional Losses}

In this section we study the distribution of aggregate portfolio losses $L_{n}$ in the general case, i.e. without a particular assumption on the structure of the conditional distribution $M_{r}$. 


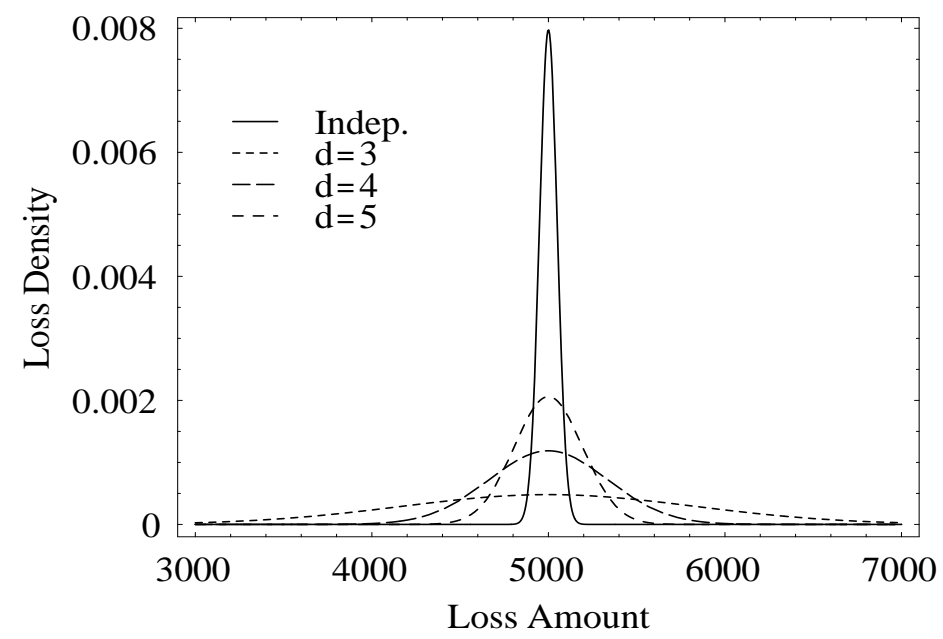

Figure 4: Approximate density of portfolio losses, varying the degree $d$ of connectedness $(r=10.000$ and $\rho=0.5)$.

In a fist step we consider the average losses in the portfolio $\Lambda_{n}$. Let $\mu=\int_{0}^{1} \nu_{\rho} Q(d \rho)$ again be an equilibrium rating distribution where the average number of low-rated firms in the whole economy is distributed according to $Q$. The joint distribution of losses is given by the mixture

$$
\beta(d w)=\int\left(\otimes_{i \in \mathbb{Z}^{d}} M_{\xi(i)}\right)(d w) \mu(d \xi), \quad w \in \mathbb{R}^{\mathbb{Z}^{d}} .
$$

As with deterministic conditional losses, by a law of large numbers we have

$$
\lim _{n \rightarrow \infty} \frac{L_{n}}{\left|\Lambda_{n}\right|}=\rho\left(l_{1}-l_{0}\right)+l_{0}
$$

$\beta$-almost surely. $\rho$ is random with distribution $Q$ governing the empirical average of low-rated firms in the economy. Due to the ergodicity of the measures $\nu_{\rho}$, in infinite portfolios average losses do not depend on the interaction of firms. Our next result shows that in large portfolios the quantiles $q_{\alpha}\left(L_{n}\right)$ of the loss distribution are essentially governed by the quantiles of $Q$.

Proposition 4.3. Let $q_{\alpha}(Q)$ be the $\alpha$-quantile of the distribution $Q$ and assume that the cumulative distribution function $G$ of $Q$ is strictly increasing at $q_{\alpha}(Q)$, i.e. $G\left(q_{\alpha}(Q)+\epsilon\right)>\alpha$ and $G\left(q_{\alpha}(Q)-\epsilon\right)<\alpha$ for every $\epsilon>0$. Then

$$
\lim _{n \rightarrow \infty} \frac{q_{\alpha}\left(L_{n}\right)}{\left|\Lambda_{n}\right|}=q_{\alpha}(Q)\left(l_{1}-l_{0}\right)+l_{0} .
$$

where $l_{r}$ is the expected loss on a position with a firm in rating $r \in\{0,1\}$. 


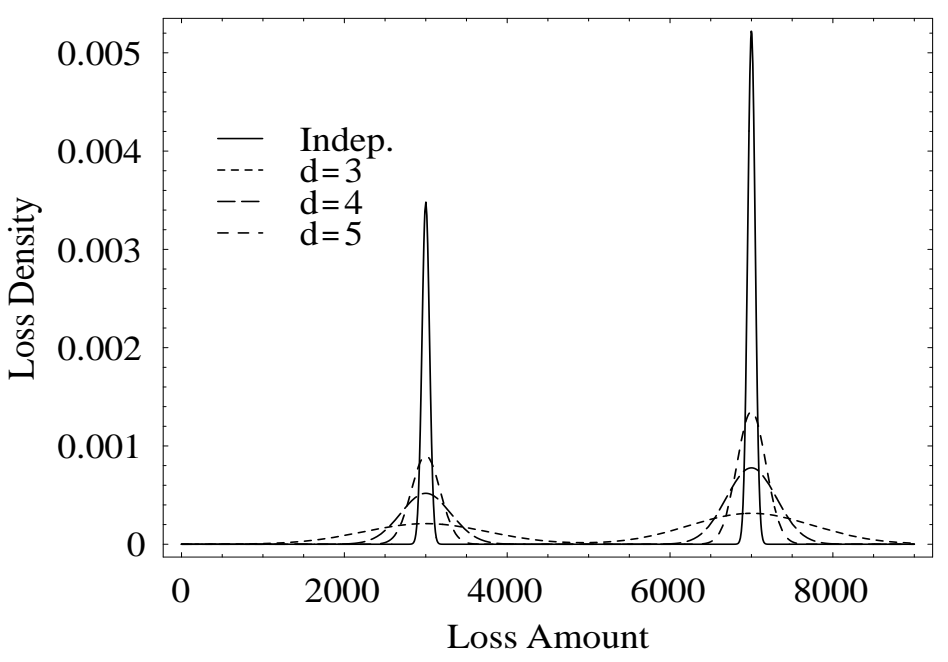

Figure 5: Approximate density of portfolio losses, varying the degree $d$ of connectedness $\left(r=10.000\right.$ and $Q=0.4 \delta_{0.3}+$ $\left.0.6 \delta_{0.7}\right)$.

Frey \& McNeil (2001) proved a similar result for exchangeable Bernoulli mixture models, in which credit losses are conditionally independent given some exogenous macro-economic factors. In this context the quantiles of the given factor distribution (the mixing distribution) essentially determine the quantiles of the loss distribution for large homogeneous portfolios. This has immediate consequences for the degree of model risk in Bernoulli mixture models: the tail properties of the chosen mixing distribution govern the tail properties of the loss distribution. This very tail behavior is of central significance for risk measurement and management, as it corresponds to a probabilistic assessment of the scenarios with extremely large losses. Analogously, in our credit contagion approach the tail properties of $Q$ essentially govern the tail behavior of aggregate losses in large portfolios, i.e. the extent of excessive fluctuations of the losses $L_{\infty}$ in an infinitely large portfolio. Here model risk is associated with the distribution $Q$.

Let us now again first focus on the case $Q=\delta_{\rho}$ for $\rho \in(0,1)$, and investigate the distribution of the losses

$$
\beta(d w)=\int\left(\otimes_{i \in \mathbb{Z}^{d}} M_{\xi(i)}\right)(d w) \nu_{\rho}(d \xi), \quad w \in \mathbb{R}^{\mathbb{Z}^{d}}
$$

Like in the case of deterministic conditional losses, for large portfolios the law of the losses $L_{n}$ can again be approximated by a normal distribution. For the 
expected loss we introduce the following notation:

$$
m=(1-\rho) \cdot l_{0}+\rho \cdot l_{1} .
$$

From Theorem 4.1 we can derive the weak convergence of the losses in the stochastic case:

Theorem 4.4. Let $d>2$ and suppose that $Q=\delta_{\rho}$ for $\rho \in(0,1)$. For large portfolios the distribution of losses satisfies

$$
\left|\Lambda_{n}\right|^{-\frac{d+2}{2 d}} \cdot\left(L_{n}-\left|\Lambda_{n}\right| \cdot m\right)=\left|\Lambda_{n}\right|^{-\frac{d+2}{2 d}} \cdot \sum_{i \in \Lambda_{n}}(U(i)-m) \Longrightarrow \mathcal{N}\left(0,\left(l_{1}-l_{0}\right)^{2} \cdot \sigma^{2}\right)
$$

where the $\sigma^{2}$ denotes the limiting variance (14). The loss distribution can uniformly be approximated, i.e.

$$
\sup _{x \in \mathbb{R}}\left|\beta\left(L_{n} \geq x\right)-\Phi\left(\frac{\left|\Lambda_{n}\right|^{1 / 2} m-\left|\Lambda_{n}\right|^{-1 / 2} x}{\left(l_{1}-l_{0}\right) \sigma \cdot\left|\Lambda_{n}\right|^{1 / d}}\right)\right| \leq \epsilon_{n}
$$

where $\epsilon_{n} \rightarrow 0$ as $n \rightarrow \infty$.

Based on inequality (25), interpolation between sizes of the portfolios $\Lambda_{n}$ allows us to define the approximate loss probabilities larger than $x \in \mathbb{R}_{+}$for portfolio size $r \in \mathbb{R}_{+}$by

$$
\Phi\left(\frac{r^{1 / 2} m-r^{-1 / 2} x}{\left(l_{1}-l_{0}\right) \sigma \cdot r^{1 / d}}\right) .
$$

This result corresponds to formula (18) which we obtained in the case with deterministic conditional losses. In case of stochastic conditional losses the limiting variance is multiplied by the factor $\left(l_{1}-l_{0}\right)^{2}$ depending only on the expected value of the loss distributions $M_{r}, r \in\{0,1\}$. Because of the nonclassical re-scaling the random fluctuations of the distributions $M_{r}$ are averaged out in the normal approximation.

In analogy to Corollary 4.2, let us now extend our analysis of the loss distribution to general invariant rating distributions $\mu$, which are mixtures of the extremal measures we have considered so far. The joint distribution of the losses is given by the mixture

$$
\beta(d w)=\int\left(\otimes_{i \in \mathbb{Z}^{d}} M_{\xi(i)}\right)(d w) \mu(d \xi), \quad w \in \mathbb{R}^{\mathbb{Z}^{d}} .
$$

Corollary 4.5. Let $d>2$. For a large portfolio, the distribution of losses $L_{n}$ can uniformly be approximated:

$$
\sup _{x \in \mathbb{R}}\left|\beta\left(L_{n} \geq x\right)-\int \Phi\left(\frac{\left|\Lambda_{n}\right|^{1 / 2} m-\left|\Lambda_{n}\right|^{-1 / 2} x}{\left(l_{1}-l_{0}\right) \sigma(\rho)\left|\Lambda_{n}\right|^{1 / d}}\right) Q(d \rho)\right| \leq \epsilon_{n},
$$

where $m=l_{0} \cdot(1-\rho)+l_{1} \cdot \rho$ and $\epsilon_{n} \rightarrow 0$ as $n \rightarrow \infty$. 
Compared with inequality (23), the approximate variance $\sigma^{2}(\rho)$ is multiplied by a factor $\left(l_{1}-l_{0}\right)^{2}$ and the averages of low ratings $\rho$ are replaced by $m$, if conditional losses are stochastic. Qualitatively, the approximate loss distributions behave very similar in both cases (23) and (26). It is interesting to observe that the fluctuations of the distributions $M_{r}$ around their means are averaged out in the normal approximation; only the expectations enter the inequality (26).

\section{Conclusion}

A thorough understanding of contagious credit quality deterioration, which drives aggregated loss risk of portfolios of financial positions, is essential for the regulation of financial institutions and the control of systemic risk in financial markets. We model credit contagion phenomena and study the distribution of aggregate credit losses on large financial portfolios.

The joint dynamics of firms' credit ratings is modeled by a voter process. We investigate the long-run behavior of the rating process, and find that the structure of the equilibrium rating distribution depends on the complexity of the inter-firm connectivity structure of the economy. While with a low degree of connectivity the long-run rating behavior is trivial, with a high connectivity degree this behavior becomes rich and complex. With increasing connectivity the degree of systemic risk decreases. We further analyze the equilibrium rating distribution arising in a large homogeneous economy with a complex connectivity structure. We find that the ratings are governed by a mixture of extremal distributions. The mixing distribution corresponds to the distribution of the average number of low-rated firms in the whole economy, which is a random quantity.

Considering losses on a portfolio of positions contracted with firms subject to credit contagion, we find that asymptotically not all loss uncertainty averages out. Due to the randomness of the average proportion of low-rated firms, average portfolio losses are asymptotically uncertain and governed by the mixing distribution. We show that the mixing distribution essentially governs the quantiles of the loss distribution.

As the key to the measurement and management of a portfolio's aggregated credit risk, we provide an explicit normal approximation of the loss distribution of a large finite portfolio. As expected, this distribution exhibits large fluctuations and 'heavy tails' when firms are interdependent and credit contagion phenomena are present. 


\section{A Ergodic Decomposition}

This appendix provides supplementary results and a proof of Theorem 3.1.

Let $X=\{0,1\}^{\mathbb{Z}^{d}}$. By $\mathcal{M}_{e}$ we denote the space of ergodic probability measures on $X$ endowed with weak topology. Let $\mathcal{G}$ be the Borel $\sigma$-algebra on $\mathcal{M}_{e}$. We write $\mathcal{M}_{e, \rho}$ for the subspace of $\mathcal{M}_{e}$ of probability measures $\nu$ with $\nu\{\xi: \xi(0)=1\}=\rho \in[0,1]$. The theorem of Choquet states that any shiftinvariant probability measure on $X$ can be represented as a mixture of ergodic measures [Georgii (1988)]:

Theorem A.1. Let $\mu$ be a translation invariant probability measure on $X$. Then there exists a probability measure $\gamma$ on $\mathcal{M}_{e}$ such that $\mu=\int_{\mathcal{M}_{e}} \nu \gamma(d \nu)$, i.e. for all continuous functions $f \in C(X)$ it holds that $\mu(f)=\int_{\mathcal{M}_{e}} \nu(f) \gamma(d \nu)$.

The following theorem refines the statement of Choquet:

Theorem A.2. Let $\mu$ be a translation invariant probability measure on $X$. Then there exists a probability measure $Q$ on $[0,1]$ and a kernel

$$
\gamma \cdot(\cdot): \begin{cases}\mathcal{G} \times[0,1] & \rightarrow[0,1] \\ (A, \rho) & \mapsto \gamma_{\rho}(A)\end{cases}
$$

with $\gamma_{\rho}\left(\mathcal{M}_{e, \rho}\right)=1$ such that

$$
\mu=\int_{[0,1]}\left(\int_{\mathcal{M}_{e}} \nu \gamma_{\rho}(d \nu)\right) Q(d \rho) .
$$

$Q$ has the cumulative distribution function $G$ given by

$$
G(\rho)=\gamma\{\nu: \nu\{\xi: \xi(0)=1\} \leq \rho\} .
$$

Proof of Theorem A.2. For $\nu \in \mathcal{M}_{e}$ define $Y(\nu):=\nu\{\xi: \xi(0)=1\} . Y$ is measurable, since $Y$ is continuous. Define $\mathcal{F}:=\sigma(Y)$. Let

$$
\gamma(\cdot \mid \mathcal{F})(\cdot): \begin{cases}\mathcal{M}_{e} \times \mathcal{G} & \rightarrow[0,1] \\ (\nu, A) & \mapsto \gamma(A \mid \mathcal{F})(\nu)\end{cases}
$$

be a regular version of the conditional probability, i.e. for fixed $A \in G$ the random variable $\gamma(A \mid \mathcal{F})$ is $\mathcal{G}$-measurable, and for fixed $\nu \in \mathcal{M}_{e} \gamma(\cdot \mid \mathcal{F})(\nu)$ is a probability measure.

For $A \in \mathcal{G}, \gamma(A \mid \mathcal{F})$ is $\sigma(Y)$-measurable. By Doob's functional representation theorem there exists a measurable mapping $\gamma \cdot(A):[0,1] \rightarrow[0,1]$ such that

$$
\gamma(A \mid \mathcal{F})(\nu)=\gamma_{Y(\nu)}(A)
$$


Since $\gamma(\cdot \mid \mathcal{F})(\cdot)$ is regular, it follows that $\gamma(\cdot)$ is a kernel.

For $C \in \mathcal{G}$ we have

$$
\begin{aligned}
\gamma(C) & =\int_{\mathcal{M}_{e}} \gamma(C \mid \mathcal{F})(\nu) \gamma(d \nu)=\int_{\mathcal{M}_{e}} \gamma_{Y(\nu)}(C) \gamma(d \nu) \\
& =\int_{[0,1]} \gamma_{\rho}(C) Q(d \rho)
\end{aligned}
$$

where $Q=\mathcal{L}(Y ; \gamma)=\gamma \circ Y^{-1}$

This implies for $f \in C(X)$ :

$$
\mu(f)=\int_{\mathcal{M}_{e}} \nu(f) \gamma(d \nu)=\int_{[0,1]}\left(\int_{\mathcal{M}_{e}} \nu(f) \gamma_{\rho}(d \nu)\right) Q(d \rho) .
$$

Note that for $\rho \in[0,1] \mathcal{M}_{e, \rho}$ is measurable, since it is a closed set in $\mathcal{M}_{e}$. Then for any $\nu \in \mathcal{M}_{e, \rho}$ :

$$
\gamma_{\rho}\left(\mathcal{M}_{e, \rho}\right)=\gamma_{Y(\nu)}\left(\mathcal{M}_{e, \rho}\right)=\gamma\left(\mathcal{M}_{e, \rho} \mid \mathcal{F}\right)(\nu)
$$

Observe now that

$$
\int_{\mathcal{M}_{e, \rho}} \gamma\left(\mathcal{M}_{e, \rho} \mid \mathcal{F}\right)(\nu) \gamma(d \nu)=\gamma\left(\mathcal{M}_{e, \rho}\right)=\int_{\mathcal{M}_{e, \rho}} \gamma(d \nu)
$$

Hence, $\gamma_{\rho}\left(\mathcal{M}_{e, \rho}\right)=1$.

Finally, we have to show that $Q$ has the cumulative distribution function $G$ :

$$
Q((-\infty, \rho])=\gamma(Y \leq \rho)=\gamma\{\nu: \nu\{\xi: \xi(0)=1\} \leq \rho\}=G(\rho) .
$$

This completes the proof.

Having proved this refined decomposition, we are now in a position to prove Theorem 3.1 in the text:

Proof of Theorem 3.1. Let $f \in C(X)$. Writing $\mu_{t}^{\xi}$ instead of $\mu_{t}^{\delta_{\xi}}$, we have that

$$
\begin{aligned}
\mu_{t}^{\kappa}(f) & =\int \mu_{t}^{\xi}(f) \kappa(d \xi) \\
& =\int_{0}^{1}\left(\int_{\mathcal{M}_{e}}\left(\int \mu_{t}^{\xi}(f) \nu(d \xi)\right) \gamma_{\rho}(d \nu)\right) Q(d \rho) \\
& =\int_{0}^{1}\left(\int_{\mathcal{M}_{e}} \mu_{t}^{\nu}(f) \gamma_{\rho}(d \nu)\right) Q(d \rho)
\end{aligned}
$$


Moreover, by the bounded convergence theorem

$$
\begin{aligned}
\lim _{t \rightarrow \infty} \mu_{t}^{\kappa}(f) & =\lim _{t \rightarrow \infty} \int_{0}^{1}\left(\int_{\mathcal{M}_{e}} \mu_{t}^{\nu}(f) \gamma_{\rho}(d \nu)\right) Q(d \rho) \\
& =\int_{0}^{1}\left(\int_{\mathcal{M}_{e}}\left(\lim _{t \rightarrow \infty} \mu_{t}^{\nu}(f)\right) \gamma_{\rho}(d \nu)\right) Q(d \rho)
\end{aligned}
$$

since $\left|\mu_{t}^{\nu}(f)\right| \leq\|f\|_{\infty}<\infty$. Noting that $\lim _{t \rightarrow \infty} \mu_{t}^{\nu}(f)=\nu_{\rho}(f)$ on $\mathcal{M}_{e, \rho}$ and that $\gamma_{\rho}\left(\mathcal{M}_{e, \rho}\right)=1$, we have

$$
\lim _{t \rightarrow \infty} \mu_{t}^{\kappa}(f)=\int_{0}^{1} \nu_{\rho}(f) Q(d \rho),
$$

which is our assertion.

\section{B Normal Approximation}

This appendix is devoted to the proof of Theorems 4.1 and 4.4 as well as Corollary 4.2. We start by considering the escape probability $\gamma_{d}$.

Theorem B.1. Let $Y_{n}$ be a simple random walk on $\mathbb{Z}^{d}$ with $d \geq 3$. The escape probability $\gamma_{d}$ can be calculated by

$$
\gamma_{d}=\frac{1}{J(d)}
$$

where the quantity $J(d)$ is defined by

$$
J(d)=(2 \pi)^{-d} \int_{(-\pi, \pi)^{d}}\left(1-\frac{1}{d} \sum_{m=1}^{d} \cos x_{m}\right)^{-1} d x .
$$

Numerical values are given in the following table:

\begin{tabular}{lll}
$d$ & $J(d)$ & $\gamma_{d}$ \\
\hline 3 & 1.516386 & 0.659463 \\
4 & 1.239467 & 0.806798 \\
5 & 1.156308 & 0.864821 \\
6 & 1.116963 & 0.895285 \\
7 & 1.093906 & 0.914155 \\
8 & 1.078647 & 0.927087 \\
9 & 1.067746 & 0.936552
\end{tabular}


Proof of Theorem B.1. The proof uses arguments from Chapter 3 of Durrett (1996). We denote by $\tau_{n}$ the optional time of the $n$th return of $Y_{n}$ to 0 . Note that $P\left(\tau_{1}<\infty\right)<1$ by the transience of the random walk. Hence, we have

$$
\begin{aligned}
& \sum_{m=0}^{\infty} P\left(Y_{m}=0\right)=\sum_{n=0}^{\infty} P\left(\tau_{n}<\infty\right) \\
= & \sum_{n=0}^{\infty} P\left(\tau_{1}<\infty\right)^{n}=\frac{1}{1-P\left(\tau_{1}<\infty\right)}=\frac{1}{\gamma_{d}} .
\end{aligned}
$$

Thus,

$$
\gamma_{d}=\left(\sum_{m=0}^{\infty} P\left(Y_{n}=0\right)\right)^{-1}
$$

It remains to be shown that

$$
\sum_{m=0}^{\infty} P\left(Y_{n}=0\right)=J(d)
$$

By $\phi$ we denote the characteristic function of one step of the random walk, i.e.

$$
\phi(x)=E\left(\exp \left(i x Y_{1}\right)\right)=\frac{1}{d} \sum_{j=1}^{d} \cos x_{j}
$$

where the last identity follows from Euler's formula. The independence of the increments of the random walk implies that

$$
\phi^{n}(x)=E\left(\exp \left(i x Y_{n}\right)\right)
$$

Since $Y_{n}$ is $\mathbb{Z}^{d}$-valued, we have the following simple Fourier inversion:

$$
P\left(Y_{n}=0\right)=(2 \pi)^{-d} \int_{(-\pi, \pi)^{d}} \phi^{n}(x) d x
$$

For $r \in(0,1)$, it holds that

$$
\left|\sum_{n=0}^{\infty} r^{n} \phi^{n}(x)\right| \leq \sum_{n=0}^{\infty} r^{n}\|\phi\|_{\infty}^{n}=\sum_{n=0}^{\infty} r^{n}=\frac{1}{1-r}
$$

By the bounded convergence theorem, we get

$$
\sum_{n=0}^{\infty} r^{n} P\left(Y_{n}=0\right)=(2 \pi)^{-d} \int_{(-\pi, \pi)^{d}} \frac{1}{1-r \phi(x)} d x
$$


Observe that $\phi$ is a real function. The term

$$
\frac{1}{1-r \phi(x)}
$$

is bounded between 0 and 1 if $\phi(x) \leq 0$, and increases to $(1-\phi(x))^{-1}$ as $r \nearrow 1$ if $\phi(x)>0$. Hence, the monotone and bounded convergence theorem imply that

$$
\sum_{n=0}^{\infty} P\left(Y_{n}=0\right)=(2 \pi)^{-d} \int_{(-\pi, \pi)^{d}}\left(1-\frac{1}{d} \sum_{m=1}^{d} \cos x_{m}\right)^{-1} d x
$$

In order to obtain the numerical values in the table observe that

$$
J(d)=d \cdot I(d ; 1)=L(d ; 1)+1
$$

where the functions $I$ and $L$ are defined and evaluated in Kondo \& Hara (1987).

Proof of Theorem 4.1. From Theorem 1 in Zähle (2001) we can derive the following normal approximation result for the basic voter model in dimension $d>2$ : Let $p(i)$ be the transition probability of the first step of a simple random walk starting at 0 , i.e. $p(i)=(2 d)^{-1}$ if $i= \pm e_{j}$ where $e_{j}$ is the $j$ th unit vector, $j=1,2, \ldots, d$. Let $Z=\left(Z_{1}, \ldots, Z_{d}\right)$ be a random vector distributed according to $p$. The second moments of $Z$ are given by

$$
Q_{l, k}=E\left[Z_{l} Z_{k}\right]=\frac{1}{d} \delta_{l, k}
$$

where $\delta_{l, k}$ denotes the Kronecker symbol. Let $Q=\left(Q_{l, k}\right)$ be the matrix of the second moments, and $|Q|$ the determinate of $Q$. Since $Q$ is invertible, we can define the quadratic form

$$
\bar{Q}(x)=x^{T} Q^{-1} x .
$$

Denoting the identity matrix in $\mathbb{R}^{d \times d}$ by $I$, we get $Q=\frac{1}{d} I, Q^{-1}=d \cdot I$, and $|Q|=d^{-d}$.

From a general result of Zähle (2001) for the linear voter model it follows in the particular case of the basic voter model for any Schwartz function $\phi$ : $\mathbb{R}^{d} \rightarrow \mathbb{R}$ that as $r \rightarrow \infty$

$$
r^{-(d+2) / 2} \sum_{i \in \mathbb{Z}^{d}}[\xi(i)-\rho] \cdot \phi\left(\frac{i}{r}\right) \Rightarrow \mathcal{N}\left(0, C_{\rho} B(\phi, \phi)\right) .
$$

Here $B$ is the bilinear functional on the Schwartz space $\mathcal{S}$ given by

$$
B(\phi, \psi)=\int_{\mathbb{R}^{d}} \int_{\mathbb{R}^{d}} \frac{\phi(x) \psi(y)}{\bar{Q}(x-y)^{(d-2) / 2}} d x d y .
$$


The multiplicative constant $C_{\rho}$ is defined by

$$
C_{\rho}=\rho(1-\rho) \frac{\gamma}{2 \pi^{d / 2}|Q|^{1 / 2}} \Gamma\left(\frac{d-2}{2}\right)
$$

where $\Gamma$ denotes the Gamma function and $\gamma$ is the escape probability of a discrete time simple random walk in dimension $d$ which starts in 0 . It can be shown that the result holds also if $\phi$ is chosen to be the indicator of a box, e.g.

$$
\phi=1_{[-1,1]^{d}}
$$

Hence as $n \rightarrow \infty$ we get weak convergence,

$$
\begin{aligned}
& \left|\Lambda_{n}\right|^{-\frac{d+2}{2 d}} \sum_{i \in \Lambda_{n}}(\xi(i)-\rho)=(2 n+1)^{-\frac{d+2}{2}} \sum_{i \in \mathbb{Z}^{d}}(\xi(i)-\rho) 1_{[-1,1]^{d}}\left(\frac{i}{n}\right) \\
\Rightarrow & \mathcal{N}\left(0, \frac{C_{\rho} B\left(1_{[-1,1]^{d}}, 1_{[-1,1]^{d}}\right)}{2^{d+2}}\right) .
\end{aligned}
$$

In order to verify the approximation result (13), we have to calculate the asymptotic variance. It follows that

$$
\begin{aligned}
& B\left(1_{[-1,1]^{d}}, 1_{[-1,1]^{d}}\right)=\frac{1}{d^{(d-2) / 2}} \int_{[-1,1]^{d}} \int_{[-1,1]^{d}}\|x-y\|_{2}^{-(d-2)} d x d y, \\
& C_{\rho}=\rho(1-\rho) \frac{\gamma}{2 \pi^{d / 2} \cdot d^{-d / 2}} \cdot \Gamma\left(\frac{d-2}{2}\right) .
\end{aligned}
$$

It is now easy to see that

$$
\sigma^{2}=\frac{C_{\rho} B\left(1_{[-1,1]^{d}}, 1_{[-1,1]^{d}}\right)}{2^{d+2}}
$$

which proves (14).

Next we derive the uniform approximation (16). Since the distribution function of the normal distribution is continuous, it follows from Exercise 2.6. in Chapter 2 of Durrett (1996) that

$$
\sup _{z \in \mathbb{R}}\left|\nu_{\rho}\left(\frac{\left|\Lambda_{n}\right|^{-\frac{d+2}{2 d}}\left(L_{n}-\left|\Lambda_{n}\right| \rho\right)}{\sigma} \geq z\right)-\Phi(-z)\right| \leq \epsilon_{n}
$$

where $\epsilon_{n} \rightarrow 0$ as $n \rightarrow \infty$. Approximation (16) follows immediately, since

$$
\left\{L_{n} \geq x\right\}=\left\{\frac{\left|\Lambda_{n}\right|^{-\frac{d+2}{2 d}}\left(L_{n}-\left|\Lambda_{n}\right| \rho\right)}{\sigma} \geq \frac{\left|\Lambda_{n}\right|^{-\frac{d+2}{2 d}}\left(x-\left|\Lambda_{n}\right| \rho\right)}{\sigma}\right\} .
$$


Finally, rewrite the last term in the last bracket:

$$
\frac{\left|\Lambda_{n}\right|^{-\frac{d+2}{2 d}}\left(x-\left|\Lambda_{n}\right| \rho\right)}{\sigma}=\frac{\left|\Lambda_{n}\right|^{-1 / 2} x-\left|\Lambda_{n}\right|^{1 / 2} \rho}{\sigma \cdot\left|\Lambda_{n}\right|^{1 / d}} .
$$

The escape probability $\gamma=\gamma_{d}$ is calculated by Theorem B.1.

Proof of Corollary 4.2. The distribution of

$$
\left|\Lambda_{n}\right|^{-\frac{d+2}{2 d}} \sum_{i \in \Lambda_{n}}(\xi(i)-\rho)
$$

under the measure $\nu_{\rho}$ will be denoted by $\varsigma_{\rho}^{n}$. We define the quantity

$$
\delta_{\rho}^{n}:=\sup _{n^{\prime} \geq n} \sup _{z \in \mathbb{R}}\left|\varsigma_{\rho}^{n^{\prime}}([z, \infty))-\Phi\left(-\frac{z}{\sigma(\rho)}\right)\right| .
$$

Inequality (16) implies that $\delta_{\rho}^{n}$ converges to 0 for all $\rho \in(0,1)$ as $n \rightarrow \infty$. Observe that $\rho \mapsto \delta_{\rho}^{n}$ is measurable. For $\epsilon>0$ we can therefore define measurable sets

$$
A_{\epsilon}^{n}=\left\{\rho \in(0,1): \delta_{\rho}^{n}<\epsilon\right\} .
$$

Then $A_{\epsilon}^{n} \subseteq A_{\epsilon}^{n+1}$, and $Q\left(A_{\epsilon}^{n}\right) \nearrow 1$ as $n \rightarrow \infty$. Choose $n_{0}$ large enough such that

$$
Q\left(A_{\epsilon}^{n_{0}}\right) \geq 1-\epsilon
$$

Let $\rho \mapsto z(\rho)$ be a measurable mapping. Then for all $n \geq n_{0}$ we get

$$
\begin{aligned}
& \left|\int\left[\varsigma_{\rho}^{n}([z(\rho), \infty))-\Phi\left(-\frac{z(\rho)}{\sigma(\rho)}\right)\right] Q(d \rho)\right| \\
\leq & 2\left(1-Q\left(A_{\epsilon}^{n}\right)\right)+\sup _{\rho \in A_{\epsilon}^{n}} \sup _{z^{\prime} \in \mathbb{R}}\left|\varsigma_{\rho}^{n}\left(\left[z^{\prime}, \infty\right)\right)-\Phi\left(-\frac{z^{\prime}}{\sigma(\rho)}\right)\right| \\
\leq & 3 \epsilon
\end{aligned}
$$

Let $x \in \mathbb{R}$ be arbitrary, and let $n \geq n_{0}$. We can choose

$$
z(\rho)=\left|\Lambda_{n}\right|^{-\frac{d+2}{2 d}}\left(x-\left|\Lambda_{n}\right| \rho\right) .
$$

It follows that for any $x \in \mathbb{R}$ and $n \geq n_{0}$ the following inequality holds

$$
\left|\int \nu_{\rho}\left(L_{n} \geq x\right) Q(d \rho)-\int \Phi\left(\frac{\left|\Lambda_{n}\right|^{1 / 2} \rho-\left|\Lambda_{n}\right|^{-1 / 2} x}{\sigma(\rho)\left|\Lambda_{n}\right|^{1 / d}}\right) Q(d \rho)\right| \leq 3 \epsilon .
$$

Proof of TheOrem 4.4. This is a corollary of the normal approximation results in the deterministic case. Define the function $f:\{0,1\} \rightarrow\left\{l_{0}, l_{1}\right\}$ 
by $f(0)=l_{0}$ and $f(1)=l_{1} \cdot f$ is used to introduce the random variables $m_{i}=f(\xi(i)), i \in \mathbb{Z}^{d}$. It is easy to see that (13) implies

$$
\left|\Lambda_{n}\right|^{-\frac{d+2}{2 d}} \sum_{i \in \Lambda_{n}}\left(m_{i}-m\right) \Rightarrow \mathcal{N}\left(0,\left(l_{1}-l_{0}\right)^{2} \cdot \sigma^{2}\right)
$$

Denote now by $\left(X_{r, i}\right)_{i \in \mathbb{Z}^{d}}$ independent random variables with distribution $M_{r}$, $r \in\{0,1\}$. Then we can rewrite the renormalized losses as

$$
\begin{aligned}
\left|\Lambda_{n}\right|^{-\frac{d+2}{2 d}}\left(L_{n}-\left|\Lambda_{n}\right| m\right) & =\left|\Lambda_{n}\right|^{-\frac{d+2}{2 d}} \sum_{i \in \Lambda_{n}, \xi(i)=0}\left(X_{0, i}-m_{0}\right) \\
& +\left|\Lambda_{n}\right|^{-\frac{d+2}{2 d}} \sum_{i \in \Lambda_{n}, \xi(i)=1}\left(X_{1, i}-m_{1}\right) \\
& +\left|\Lambda_{n}\right|^{-\frac{d+2}{2 d}} \sum_{i \in \Lambda_{n}}\left(m_{i}-m\right)
\end{aligned}
$$

The last summand on the right hand side converges weakly according to (31). The other two terms converge almost surely to 0; w.l.o.g. we will prove this fact only for the first term, i.e.

$$
\left|\Lambda_{n}\right|^{-\frac{d+2}{2 d}} \sum_{i \in \Lambda_{n}, \xi(i)=0}\left(X_{0, i}-m_{0}\right)=\left|\Lambda_{n}\right|^{-\frac{d+2}{2 d}} \sum_{i \in \Lambda_{n}, \xi(i)=0}\left(X_{0, i}-l_{0}\right) .
$$

The random number of summands in (32) equals $c(n)=\left|\left\{i \in \Lambda_{n}: \xi(i)=0\right\}\right|$ and is almost surely increasing to $\infty$ as $n \rightarrow \infty$. Theorem 8.7. of Chapter 1 in Durrett (1996) implies for $\epsilon>0$ that

$$
c(n)^{-1 / 2}(\log c(n))^{-(1 / 2+\epsilon)} \sum_{i \in \Lambda_{n}, \xi(i)=0}\left(X_{0, i}-l_{0}\right)
$$

converges to 0 as $n \rightarrow \infty$. The last result can also be viewed as a consequence of the law of iterated logarithm.

Now observe that for $\epsilon>0$ the sequence $c(n)$ satisfies

$$
\frac{c(n)^{1 / 2}(\log c(n))^{1 / 2+\epsilon}}{\left|\Lambda_{n}\right|^{\frac{d+2}{2 d}}} \leq \frac{\left|\Lambda_{n}\right|^{1 / 2}\left(\log \left|\Lambda_{n}\right|\right)^{1 / 2+\epsilon}}{\left|\Lambda_{n}\right|^{\frac{d+2}{2 d}}}=\frac{\left(\log \left|\Lambda_{n}\right|\right)^{1 / 2+\epsilon}}{\left|\Lambda_{n}\right|^{1 / d}} .
$$

The last term converges to 0 as $n \rightarrow \infty$. This fact together with (33) implies that the terms in (32) converge to 0 as $n \rightarrow \infty$.

Altogether we obtain for $n \rightarrow \infty$ the weak convergence,

$$
\left|\Lambda_{n}\right|^{-\frac{d+2}{2 d}} \cdot\left(L_{n}-\left|\Lambda_{n}\right| \cdot m\right) \Rightarrow \mathcal{N}\left(0,\left(l_{1}-l_{0}\right)^{2} \cdot \sigma^{2}\right) .
$$

The uniform approximation (25) is obtained with the same arguments as in the deterministic case.

Proof of Corollary 4.5. Analogous to the proof of Corollary 4.2. 


\section{Loss Quantiles}

Proof of Proposition 4.3. First observe that, due to (24),

$$
\lim _{n \rightarrow \infty} \nu_{\rho}\left(\frac{\frac{L_{n}}{\left|\Lambda_{n}\right|}-l_{0}}{l_{1}-l_{0}} \leq a\right)= \begin{cases}1 & , \quad \rho<a \\ 0, & \rho>a\end{cases}
$$

Let $\epsilon>0$ and let $G$ be the cumulative distribution function of $Q$. Then

$$
\begin{aligned}
& \limsup _{n \rightarrow \infty} \mu\left\{L_{n}-l_{0}\left|\Lambda_{n}\right| \leq\left|\Lambda_{n}\right|\left(l_{1}-l_{0}\right)\left(q_{\alpha}(Q)-\epsilon\right)\right\} \\
& =\limsup _{n \rightarrow \infty} \int_{0}^{1} \nu_{\rho}\left\{L_{n}-l_{0}\left|\Lambda_{n}\right| \leq\left|\Lambda_{n}\right|\left(l_{1}-l_{0}\right)\left(q_{\alpha}(Q)-\epsilon\right)\right\} d G(\rho) \\
& \leq \int_{0}^{1} \limsup _{n \rightarrow \infty} \nu_{\rho}\left(\frac{\frac{L_{n}}{\left|\Lambda_{n}\right|}-l_{0}}{l_{1}-l_{0}} \leq q_{\alpha}(Q)-\epsilon\right) d G(\rho) \\
& \leq \int_{0}^{1} 1_{\left(-\infty, q_{\alpha}(Q)-\epsilon\right]}(\rho) d G(\rho) \\
& =G\left(q_{\alpha}(Q)-\epsilon\right)<\alpha,
\end{aligned}
$$

where the last equality is strict by assumption. The first inequality follows from Fatou's lemma. Analogously,

$$
\liminf _{n \rightarrow \infty} \mu\left\{L_{n}-l_{0}\left|\Lambda_{n}\right| \leq\left|\Lambda_{n}\right|\left(l_{1}-l_{0}\right)\left(q_{\alpha}(Q)+\epsilon\right)\right\} \geq G\left(q_{\alpha}(Q)+\epsilon\right)>\alpha .
$$

Hence, for $n$ large enough:

$$
\left|\Lambda_{n}\right|\left(l_{1}-l_{0}\right)\left(q_{\alpha}(Q)-\epsilon\right) \leq q_{\alpha}\left(L_{n}-l_{0}\left|\Lambda_{n}\right|\right) \leq\left|\Lambda_{n}\right|\left(l_{1}-l_{0}\right)\left(q_{\alpha}(Q)+\epsilon\right) .
$$

The claim follows from observing that $q_{\alpha}\left(L_{n}-l_{0}\left|\Lambda_{n}\right|\right)=q_{\alpha}\left(L_{n}\right)-l_{0}\left|\Lambda_{n}\right|$.

\section{References}

Allen, Franklin \& Douglas Gale (2000), 'Financial contagion', Journal of Political Economy 108, 1-33.

CSFP (1997), CreditRisk+: A credit risk management framework. Technical Document, London.

Davis, Mark \& Violet Lo (2001), Modeling default correlation in bond portfolios, in C.Alexander, ed., 'Mastering Risk Volume 2: Applications', Financial Times Prentice Hall, pp. 141-151. 
Dembo, Amir, Jean-Dominique Deuschel \& Darrell Duffie (2002), Large portfolio losses. Working Paper, Stanford University.

Duffee, Gregory (1998), 'The relation between treasury yields and corporate yield spreads', Journal of Finance 53, 2225-2243.

Duffie, Darrell \& Kenneth J. Singleton (1999), 'Modeling term structures of defaultable bonds', Review of Financial Studies 12, 687-720.

Duffie, Darrell, Mark Schroder \& Costis Skiadas (1996), 'Recursive valuation of defaultable securities and the timing of resolution of uncertainty', Annals of Applied Probability 6, 1075-1090.

Durrett, Richard (1996), Probability: Theory and Examples, Duxbury Press, Belmont.

Freixas, Xavier, Bruno Parigi \& Jean-Charles Rochet (2000), 'Systemic risk, interbank relations and liquidity provision by the central bank', Journal of Money, Credit and Banking 32, 611-638.

Frey, Rüdiger \& Alexander J. McNeil (2001), Modeling dependent defaults. Working Paper, Department of Mathematics, ETH Zürich.

Georgii, Hans-Otto (1988), Gibbs Measures and Phase Transitions, Walter de Gruyter, Berlin.

Giesecke, Kay \& Stefan Weber (2002), Cyclical correlations, credit contagion, and portfolio losses. Working Paper, Humboldt-Universität zu Berlin and Technische Universität Berlin.

Giesecke, Kay (2001), Correlated default with incomplete information. Working Paper 30, SFB 373, Department of Economics, Humboldt-Universität zu Berlin.

Gupton, Gregory, Christopher Finger \& M. Bhatia (1997), CreditMetrics. Technical Document, New York.

Jarrow, Robert A., David Lando \& Stuart M. Turnbull (1997), 'A markov model of the term structure of credit risk spreads', Review of Financial Studies 10(2), 481-523.

Jarrow, Robert A. \& Fan Yu (2001), 'Counterparty risk and the pricing of defaultable securities', Journal of Finance 56(5), 555-576. 
Jarrow, Robert A. \& Stuart M. Turnbull (1995), 'Pricing derivatives on financial securities subject to credit risk', Journal of Finance 50(1), 53-86.

Kealhofer, Stephen (1998), Portfolio management of default risk. Working Paper, KMV Corp.

Keenan, Sean (2000), Historical default rates of corporate bond issuers 19201999. Moody's Investors Services.

Kiyotaki, Nobuhiro \& John Moore (1997), Credit chains. Working Paper, London School of Economics.

Kondo, Kei-ichi \& Takashi Hara (1987), 'Critical exponent of susceptibility for a class of general ferromagnets in $d>4$ dimensions', Journal of Mathematical Physics 28(5), 1206-1208.

Lando, David (1998), 'On cox processes and credit risky securities', Review of Derivatives Research 2, 99-120.

Liggett, Thomas (1985), Interacting Particle Systems, Springer-Verlag, Berlin.

Liggett, Thomas (1999), Stochastic Interacting Systems: Contact, Voter, and Exclusion Processes, Springer-Verlag, Berlin.

Rochet, Jean-Charles \& Jean Tirole (1996), 'Interbank lending and systemic risk', Journal of Money, Credit, and Banking 28, 733-762.

Schönbucher, Philipp \& Dirk Schubert (2001), Copula-dependent default risk in intensity models. Working Paper, Universität Bonn.

Zähle, Iljana (2001), 'Renormalization of the voter model in equilibrium', $A n$ nals of Probability 29(3), 1262-1302. 